\title{
CUIDADO HUMANO E TECNOLOGIA NA ENFERMAGEM CONTEMPORÂNEA E COMPLEXA
}

\author{
Maria Aparecida Baggio', Alacoque Lorenzini Erdmann², Grace Teresinha Marcon Dal Sasso ${ }^{3}$
}

\footnotetext{
${ }^{1}$ Doutoranda pelo Programa de Pós-Graduação em Enfermagem (PEN) da Universidade Federal de Santa Catarina (UFSC). Bolsista do CNPq. Santa Catarina, Brasil. E-mail: mariabaggio@yahoo.com.br

${ }^{2}$ Doutora em Enfermagem. Professora Titular do Departamento de Enfermagem da UFSC. Pesquisadora do CNPq. Santa Catarina, Brasil. E-mail: alacoque@newsite.com.br

${ }^{3}$ Doutora em Informática em Enfermagem. Professora do Departamento de Enfermagem e do PEN/UFSC. Santa Catarina, Brasil. E-mail: grace@matrix.com.br
}

RESUMO: Este artigo tem como objetivo promover reflexões sobre cuidado humano, tecnologias da informação e o pensamento complexo no cenário contemporâneo da enfermagem. O texto relaciona os principais pressupostos do paradigma da complexidade com as tecnologias da informação e o cuidado de enfermagem em suas múltiplas dimensões, destacando as relações do ser humano com tais tecnologias. Com base nos questionamentos e reflexões, deduzimos que conceber o uso das tecnologias e contextualizar sua realidade profissional para compreender a complexidade é, para a enfermagem contemporânea, um desafio complexo. Vislumbramos a possibilidade de inovar a prática do cuidado do outro de forma a corresponder às necessidades de um mundo globalizado, não ignorando a convivência entre os seres humanos, percebida como necessária e importante para a gerência de relações de cuidado saudáveis e construtivas. Abre-se para novos questionamentos na perspectiva de uma prática de cuidado tecnologicamente melhor organizada e mais avançada.

DESCRITORES: Tecnologia da informação. Saúde. Enfermagem. Cuidados de enfermagem. Serviços de enfermagem.

\section{HUMAN CARE AND TECHNOLOGY IN CONTEMPORARY AND COMPLEX NURSING}

\begin{abstract}
The objective of this article is to promote reflections concerning human care, information technologies, and complex thought in the contemporary scenario of nursing. The text relates the presupposed principles of the complexity paradigm with information technologies and nursing care in its multiple dimensions, highlighting human relationships with such technologies. From questioning and reflection, we deduce that conceiving the use of technologies and contextualizing their professional reality in order to better comprehend complexity is a complex challenge for contemporary nursing. We further point out the possibility for innovating care practices for others in a manner which corresponds to the necessities of a globalized world, not ignoring human interaction, seen as necessary and important for managing healthy and constructive care relationships. This opens up the possibility for new questions under the perspective of a more advanced and technologically better organized care practice.
\end{abstract}

DESCRIPTORS: Information technology. Health. Nursing. Nursing care. Nursing services.

\section{EL CUIDADO HUMANO Y LA TECNOLOGÍA EN LA ENFERMERÍA CONTEMPORÁNEA Y COMPLEJA}

RESUMEN: El presente artículo tiene como objetivo promover la reflexión sobre el cuidado humano, la tecnología de la información y el pensamiento complejo en el contexto contemporáneo de la enfermería. El texto relaciona los principales postulados del paradigma de la complejidad con la tecnología de la información y el cuidado de enfermería en sus múltiples dimensiones, destacando las relaciones de los seres humanos con esas tecnologías. A partir de preguntas y reflexiones, deducimos que concebir el uso de la tecnología y contextualizar su realidad profesional para comprender la complejidad es para la enfermería contemporánea un reto complejo. Imaginamos la posibilidad de innovar la práctica del cuidado como una forma de satisfacer las necesidades de un mundo globalizado, sin ignorar la coexistencia entre los seres humanos, como necesaria e importante para la gestión de las relaciones de un cuidado sano y constructivo. Este estudio se abre a nuevas preguntas en la perspectiva de una práctica de cuidado mejor organizada y más avanzada tecnológicamente.

DESCRIPTORS: Tecnología de la información. Salud. Enfermería. Atención de enfermería. Servicios de enfermería. 


\section{INTRODUÇÃO}

As Tecnologias da Informação (TIs) têm se tornado parte da vida diária das pessoas em todo o mundo. A aplicação e o uso de produtos tecnológicos, tecnologias baseadas no computador, como os sistemas de informação para o cuidado em saúde, têm se tornado um processo em permanente evolução. Avanços na informação, nas telecomunicações e na rede de tecnologias têm levado à emergência de um novo e revolucionário paradigma para o cuidado em saúde. Novas experiências e conhecimentos que transcendem as fronteiras das disciplinas tradicionais, tais como o cuidado baseado na evidência, os serviços de saúde remotos, a saúde on-line, têm gerado novos e complexos desafios na prestação do cuidado, uma vez que novas habilidades precisarão ser aprendidas com a utilização destas tecnologias. ${ }^{1}$

Diante desse cenário, optamos por abordar esta temática com o objetivo de promover reflexões que inter-relacionam cuidado humano, tecnologias da informação e o pensamento complexo no cenário contemporâneo da enfermagem.

A escolha da temática deu-se por conta da compreensão de que, nos dias atuais, tem se tornado cada vez mais desafiador aos enfermeiros prestar um cuidado consistentemente seguro ao paciente e de alta qualidade, especialmente pelo volume de informações ainda desestruturadas, heterogêneas e desintegradas, que permeiam o cuidado, além das inúmeras demandas de tempo para avaliação clínica adequada exigidas no cuidado em saúde. As TIs e a automação, se utilizadas adequadamente, podem contribuir para racionalizar e melhorar o processo de enfermagem, por auxiliar os pacientes a alcançar melhores resultados, do modo mais seguro possível e melhor apoiar os enfermeiros no cuidado aos pacientes.

As considerações aqui apresentadas acerca do objetivo proposto foram balizadas nas concepções do paradigma da complexidade e, também, nas de autores pertinentes para as reflexões. ${ }^{2-5} \mathrm{O}$ paradigma da complexidade torna possíveis as articulações e as relações necessárias para conduzir o pensamento multidimensional e complexo na integração do cuidado humano e das TIs na enfermagem contemporânea, na busca de um sentido comum e complementar para a coleta e o gerenciamento de dados, informação e conhecimento para apoiar a tomada de decisão baseada no conhecimento sobre o cuidado do paciente.

No mundo contemporâneo, a discussão que envolve a temática sobre ser humano, cuidado e tecnologia é cada vez mais conflituosa e crítica, com opiniões tanto pessimistas quanto otimistas. Dessa forma, este artigo busca inter-relacionar os principais pressupostos do paradigma da complexidade com as tecnologias da informação, bem como o cuidado de enfermagem e suas múltiplas dimensões, dentre as quais se destacam suas relações com as tecnologias da informação.

\section{O paradigma da complexidade e as tecnolo- gias de informação}

A mundialização, o "estágio atual da era planetária", 2:64 traz incontestáveis e múltiplas informações, através da era da tecnologia (telecomunicações, internet). O desenvolvimento permitiu ao ser humano ampliar a rede de comunicação e informação entre cada um e todos. Contudo, o contexto mundial e planetário sufoca as possibilidades de inteligibilidade, que parecem atrofiadas pelo modo de pensar engessado e incapaz de conceber, contextualizar e apreender a complexidade. ${ }^{3}$

As TIs e a relação com o cuidado humano em enfermagem podem ser pensadas como um desafio complexo, dados os desafios da globalidade. ${ }^{4}$ A complexidade, de fato, permeia o cenário da enfermagem quando, de um lado, alguns profissionais resistem à apropriação destas tecnologias e, de outro, outros desafiam o seu uso como ferramenta para integração entre as diversas dimensões de cuidado.

A integração das TIs ao cuidado em enfermagem é um desafio complexo e também global quando se buscam, por meio destas tecnologias, a interação, a associação, a interdependência e a inter-relação dos componentes constituintes ou relacionados direta e indiretamente ao cuidado, seja o social, seja o educativo, o afetivo, o econômico, o político, o psicológico. Contudo, como agregar inteligente e reflexivamente as TIs ao cuidado do outro sem separar/fracionar o ser e o cuidado humano em partes?

Urge a reflexão sobre o uso das tecnologias, considerando-as como possibilidade de relacionar e integrar os múltiplos saberes, fazeres e os seres humanos, cujas partes se apresentam compartimentadas, desarticuladas, separadas, fracionadas. Daí o grande desafio: desenvolver a capacidade de contextualização e integração dos saberes e fazeres, condição específica da mente humana, para aperfeiçoar e legitimar o cuidado de enfermagem com as TIs, pois não é mais possível negar, abdicar, recusar tais tecnologias em um mundo globalizado. 
Os desafios são múltiplos, se considerada a evolução do conhecimento e do desenvolvimento tecnológico. Assim, entende-se que, por detrás do desafio do global e complexo, esconde-se outro desafio: o da expansão do saber ${ }^{4} \mathrm{e}$, por que não, $\mathrm{o}$ desafio da expansão das tecnologias.

O processo de mudanças em razão das TIs provoca variação ou mesmo transformações importantes no contexto da saúde e enfermagem. As tecnologias e os conhecimentos estão em constante movimento, num processo de organização/ desorganização/reorganização pelo próprio ser humano, para a adequação e transformação da realidade que o cerceia. Por esta razão, as áreas técnicas e cognitivas apresentam-se em importante expansão, num fluxo contínuo e variado, cujas tecnologias e conhecimentos são compostos/ recompostos, modelados/remodelados, modificados e multiplicados nas diversas áreas do saber e veiculados na forma de informação.

A evolução do saber e a gigantesca proliferação de conhecimentos, por vezes, escapam ao controle humano. ${ }^{4}$ Desse modo, a acelerada produção cognitiva, quando fragmentada e restrita apenas ao uso técnico, de forma não articulada, traz preocupações.

Com base na assertiva acima, instiga-se: Como contextualizar e englobar o conhecimento científico, ser humano e tecnologias em enfermagem sem fragmentar o cuidado humano e, ao mesmo tempo, acompanhar a expansão do saber, da informação, da comunicação? Como integrar conhecimento científico, tecnologias e cuidado de enfermagem, articulando-os para o benefício do ser humano cuidado, do cuidador e da própria profissão de enfermagem sem perder as qualidades fundamentais do cuidado do outro, que são o contato, a presença, o afeto, a relação entre os seres de cuidado envolvidos?

Em resposta a essas questões, pode-se dizer que "a tecnologia não é então um paradigma de cuidado oposto ao toque humano, mas, sobretudo, um agente e um objeto deste toque. A tecnologia por si só pode ser um fator que humaniza, mesmo nas arenas mais tecnologicamente intensas de cuidado em saúde. As dualidades da tecnologia como produto e significado; matéria e significado; produto e processo repousam não em sua oposição necessária à humanização, mas, especialmente, em suas recursividades, isto é, na sua existência como ambas, força material objetiva e como uma realidade dinâmica e construída socialmente" .5:12

O uso das TIs, entendido pelo pensamento complexo como um desenvolvimento generaliza- do e múltiplo do sistema neurocerebral artificial, ${ }^{4}$ poderá proporcionar benefícios ao cuidado de enfermagem se auxiliar o profissional a relacionar o todo e as partes com base nas informações, acontecimentos, conhecimentos disponibilizados, possibilitando a relação/inter-relação/inter-retroação entre as partes e o todo. Dessa forma, poderá favorecer o pensamento unificador e a integração das partes ao todo, a partir da reunião de informações múltiplas que, interligadas, facilitam as associações/conexões/interpretações das partes para a compreensão do todo - do ser humano cuidado. Assim, poder-se-ia dizer que o ser humano seria mais bem compreendido e cuidado na sua complexidade e totalidade com o auxílio das tecnologias, se considerados tais aspectos. ${ }^{4}$

O cuidado em enfermagem e saúde ou seus processos gerenciais e ações práticas podem ser facilitados pelas tecnologias, todavia nenhuma tecnologia poderá substituir a relação e a compreensão intersubjetiva entre os seres humanos. A capacidade de empatia, de identificação, de abertura, de projeção, de generosidade e de solidariedade é expressa na relação de comunhão, de troca e de interação entre os seres. ${ }^{2,4}$

O cuidado é subjetivo e a melhor forma para a sua compreensão é a capacidade de relacionar-se com o outro, inerente ao ser humano. Contudo, as TIs podem possibilitar a expansão cognitiva e prover informação para a melhoria do fluxo de trabalhos, do desempenho profissional e da qualidade do cuidado de enfermagem. ${ }^{6}$

\section{Cuidado de enfermagem em suas múltiplas dimensões e suas relações com as TIs}

A tecnologia da informática que emerge com o advento da globalização adquiriu importância para os indivíduos, para a sociedade e também para toda uma nação e se consolidou como estratégia para controle, organização e estrutura da informação. Foi a partir da década de 1970 e 1980, com a convergência entre as novas tecnologias e a informática, que a estrutura social urbana iniciou o processo de transformação e complexificação da rede de comunicações, incitando o crescente investimento em tecnologias e recursos humanos para atender à demanda que emergia na era da comunicação por meio do processo informatizado. ${ }^{7}$

Por conseguinte, para acompanhar os desenvolvimentos social, econômico e cultural, cada profissão, dentre as quais a enfermagem, adentrou no processo tecnológico da comunicação 
e da informação. Em razão disso, "cada grande inovação em informática abriu a possibilidade de novas relações entre os homens e computadores: códigos de programação cada vez mais intuitivos, comunicação em tempo real, redes, micro, novos princípios de interfaces... É porque dizem respeito aos humanos que estas viradas na história dos artefatos informáticos nos importam". $8: 54$

A presença das TIs no cotidiano da enfermagem, principalmente no ambiente de trabalho e cuidado, atualmente, é indispensável, e o seu desenvolvimento é inevitável, necessário e importante para o desenvolvimento da profissão e do cuidado humano. Essas tecnologias, quando adequada e inteligentemente utilizadas e administradas, poderão beneficiar a prática do cuidado ao ser humano em múltiplas esferas.

Acredita-se, nesse sentido, que a enfermagem deve incorporar a tecnologia da informática para atender às necessidades da profissão e preencher lacunas existentes tanto na educação quanto na profissão, bem como promover e utilizar a tecnologia informatizada na pesquisa, no ensino, na assistência e na administração em enfermagem. ${ }^{9}$

Assim, questiona-se: Com a crescente evolução das tecnologias, como a enfermagem pode inovar sua prática e melhorar o cuidado? Que fatores, a partir da informática, podem contribuir para mudanças na prática de enfermagem atrelando tecnologia e cuidado humano seguro?

Observa-se na prática que, quando uma nova tecnologia é proposta ou inserida no âmbito de trabalho em enfermagem, as posturas, opiniões e comportamentos são distintamente manifestados pelos profissionais. Inicialmente, a tendência da maioria é perceber o manuseio de uma nova ferramenta tecnológica como mais uma atividade/ tarefa entre inúmeras que já têm a cumprir, diminuindo seu restrito tempo e assoberbando ainda mais suas tarefas cotidianas laborais, além das críticas em relação ao cuidado humano.

Os profissionais, em seu espaço social de relações, podem ampliar as redes interacionais e integrativas para o cuidado de enfermagem em suas múltiplas dimensões, utilizando as tecnologias a favor da rede no micro e macroespaço do cotidiano (pessoal e profissional). Entende-se a rede como a transgressão de fronteiras, a abertura de conexões, a multiplicidade, a flexibilidade, a transparência, a interdependência e o acesso de todos à informação - o ilimitado e o complexo. ${ }^{10}$

A rede de interações, por meio da tecnologia da comunicação e da informação - via internet - pode conectar várias pessoas ao mesmo tempo, com respostas/conteúdos/retornos imediatos e efetivos, sem deslocamento. Pode-se citar como exemplo a troca de comunicações e informações entre profissionais, pacientes e seus familiares e outros que, direta ou indiretamente, estejam relacionados e integrados no espaço virtual, considerando-se esta interação como um cuidado. A relação de interatividade apontada não se relaciona exclusivamente ao binômio ser humano-máquina, mas à interação ser humano-ser humano, mediada pelos recursos tecnológicos (neste caso o computador) e seus sistemas, que integram esta ferramenta e permitem aos usuários a comunicação e informação. ${ }^{11}$

Será, então, que o espaço virtual poderia substituir o espaço físico? Em nosso entendimento, não são substituíveis, mas o primeiro pode adicionar funcionabilidades ao segundo. $\mathrm{O}$ espaço físico e o virtual são entendidos como espaços codependentes/inter-relacionados, e os sistemas de serviços de saúde, por meio da rede digital, geram simultaneamente tendências descentralizadoras e recentralizadoras. $^{7}$

Dessa forma, são pertinentes a criação e o desenvolvimento, nos ambientes em saúde, de redes que permitam a interação entre pessoas, tecnologias e ambiente na promoção do cuidado do outro; de TIs voltadas para uma nova inter-relação espacial entre os seres de cuidado (que cuidam e são cuidados); e nova forma de organização/ implementação do cuidado pelos profissionais. É possível, com as TIs, tanto integrar diversos profissionais ao mesmo ambiente físico, compartilhando informações comuns ao cuidado do paciente, como, por exemplo, por intermédio do PEP (Prontuário Eletrônico do Paciente), quanto permitir a organização dos espaços em saúde sem proximidade física, mas interativos, integrados e inter-relacionados, cujos usuários e profissionais dos serviços de saúde possam estar conectados a um espaço comum - virtual.

A enfermagem e demais profissões da área da saúde em geral, atualmente, estão passando por um processo de mudança e reconfiguração dos seus espaços, atividades e funções. Um exemplo disso é o processo de sistematização da assistência em saúde- enfermagem, que se encontra em fase de construção, avaliação, reconstrução e reavaliação em vários ambientes de saúde. Esse processo organizativo das ações de cuidado força a relação entre as pessoas, as TIs e a informática em um cenário com objetivo comum: melhorar o cuidado em saúde das pessoas. ${ }^{12}$ 
O ambiente informatizado em saúde pode colocar em sinergia os processos complexos de relação e comunicação entre os seres deste e fora deste espaço, estabelecendo redes interativas e integrativas de informação. Não se pretende construir um mundo de ciborgues e robôs controlados e ditados pelas tecnologias. Entretanto, racional e inteligentemente, a enfermagem e demais profissionais que interagem no espaço de relações em saúde podem utilizar a tecnologia em benefício do cuidado individual e coletivo.

\section{A tecnologia e as relações humanas na con- temporaneidade}

As TIs possibilitam o atendimento das necessidades das pessoas e, se adequadamente utilizadas, são ferramentas sociais saudáveis. Para tanto, seu desenvolvimento, utilização e evolução devem ter como foco a convergência entre o desenvolvimento humano e o tecnológico. Logo, entende-se que as tecnologias são uma ferramenta para auxiliar e facilitar a relação entre os seres humanos, porém sem substituir a relação pessoa-pessoa. ${ }^{9}$

O excessivo apreço pela tecnologia, pelo consumismo e pelo tecnicismo, na atual conjuntura social, coloca os valores humanos em xeque. Ao mesmo tempo em que o homem usufrui tecnologias avançadas, as relações humanas declinam e, da mesma forma, a ética nos relacionamentos. Ainda, o cenário da modernidade torna os relacionamentos cada vez mais distantes, limitados por interesses distintos, banalizados a ponto de ignorar valores, sentimentos, emoções e desejos. ${ }^{13}$

A relação entre ser humano e tecnologia se apresenta como contexto-dependente, ou seja, o contexto e os seres são modificados pela tecnologia, ao mesmo tempo em que também a modificam. O contexto, neste caso, é entendido como o local e as condições de trabalho, efetivamente. Na enfermagem, esta relação é entendida como cautelosa. ${ }^{14}$

Sobre os diferentes posicionamentos em relação à tecnologia e ao cuidado de enfermagem, a priori, "nada, nenhuma máquina será capaz de substituir a capacidade humana de oferecer um sorriso, um toque, um olhar de carinho... Portanto, se isto é comportamento exclusivamente humano e não pode ser substituído, deve ser uma atitude inteligente, enfatizar e fortalecer tal comportamento, usando a tecnologia para atingir melhores níveis de resultado de nossas ações puramente humanas" ${ }^{6: 2}$
O avanço tecnológico, sem dúvida, é imprescindível para os profissionais da enfermagem e saúde, mas a dimensão humana deve se sobrepor aos demais interesses, sejam econômicos, sejam comerciais ou políticos. ${ }^{15}$ Dessa forma, as ações dos profissionais que se utilizam da tecnologia devem estar voltadas para o compromisso com e pela qualidade do cuidado dos pacientes e dos cuidadores dos serviços de saúde, em busca de um viver melhor.

Entendemos que as relações de cuidado em enfermagem não podem ser substituídas, mas fortalecidas com a utilização dos recursos tecnológicos pelos profissionais, se considerados a otimização e o uso racional do tempo. Assim, há o desenvolvimento de atividades preferencialmente assistenciais e uma melhor e maior integração e gerenciamento do cuidado ao cliente como um todo continuamente.

As tecnologias permitem a comunicação e informação rápida e necessária, basta estar conectado com ela. Porém, o contato e a convivência entre os seres humanos não devem ser ignorados, pois são percebidos como necessários e importantes para o estabelecimento de relações de cuidado saudáveis e construtivas.

Em nossa sociedade, algumas pessoas buscam o contato e a interação com outras, a partir do espaço virtual, por meio de máquinas, relegando a segundo plano a vida social e o contato humano, distanciando-se do mundo real e, assim, fechandose num mundo apenas virtual. Em oposição, muitas passam a ser mais sociáveis a partir do teclado de um computador do que pessoalmente. De certa forma, essa é uma preocupação inerente ao mundo de tecnologias que não pode ser ignorada.

Nesse contexto, o desenvolvimento ameaça a civilização quando degrada as relações pessoais e torna a solidão o mal oculto da modernidade. Dessa forma, a elevação dos níveis de vida pode estar ligada à degradação da qualidade de vida, assim como a multiplicação das tecnologias que permitem o contato por meio máquinas e equipamentos pode estar ligada ao empobrecimento das relações humanas interpessoais. ${ }^{3}$

\section{Enfermagem contemporânea nas suas múl- tiplas dimensões}

A enfermagem tecnicista perde espaço para outras profissões na área da saúde, algumas recém surgidas e outras que despertaram para a importância de desenvolver tecnologias próprias para o seu fortalecimento perante as demais profissões. 
Dessa forma, a enfermagem precisa enfrentar os desafios oriundos do progresso e do desenvolvimento da área da saúde e contextualizar sua própria realidade para responder aos desafios advindos da complexidade e incertezas da enfermagem brasileira na contemporaneidade.

A enfermagem necessita inovar sua prática e torná-la singular. As TIs, se utilizadas adequadamente, podem ser uma parceria de sucesso, desde que visem integrar tecnologia e cuidado humano nas suas múltiplas dimensões, conforme conota a complexidade.

Sabe-se, a partir da literatura e da prática, que o próprio processo de enfermagem, atribuição do enfermeiro assistencial, é nos dias de hoje pouco desenvolvido na sua integralidade. ${ }^{11,16-17} \mathrm{O}$ enfermeiro ocupa-se, no cotidiano de sua atividade laboral, mais de funções administrativas e gerenciais do que especificamente assistencial. ${ }^{16}$ Entende-se aqui que essas funções administrativas ou gerenciais do cuidado ou da assistência compreendem uma dimensão mais ampla do fazer o cuidado, ou do cuidar, gerenciando as múltiplas ações, ora mais técnicas interventivas, ora mais políticas e organizativas.

Em razão disso, questiona-se: Como as TIs podem ser utilizadas para melhorar o cuidado de enfermagem na sua dimensão mais ampla? Em resposta à pergunta, "as utilizações são variadas, incluindo o suporte de informação para quem presta o cuidado (cuidadores leigos, profissionais de enfermagem ou relacionados); suporte para documentação de enfermagem, utilizando terminologia padronizada; suporte à tomada de decisão e, disponibilização de guias de conduta (guidelines)" 18:214

Apropriar-se das tecnologias disponíveis e desenvolver outras novas é um dos caminhos na área da enfermagem e saúde para aproveitar as oportunidades que ora se apresentam para interagir, produzir, gerenciar e, fundamentalmente, implementar e aperfeiçoar modalidades de cuidado em sua área profissional.

O cuidado multidimensional deve ser discutido/rediscutido, avaliado/reavaliado durante todo o processo de cuidar/gerenciar/educar, auxiliado pelas TIs. A enfermagem pode se beneficiar das tecnologias no âmbito laboral, mas terá de desenvolver novas tecnologias de cuidado, que promovam a inter-relação das diversas dimensões de cuidado, não separando, mas aproximando, somando, unindo os diversos saberes e tecnologias para a integração das partes em um todo e para o conhecimento do todo através das partes.
Estudos sobre tecnologia de informação e internet relacionada à área da enfermagem, pesquisados nos idiomas inglês, português e espanhol, encontram-se publicados, na sua maioria, no primeiro idioma e em número reduzido no segundo idioma e em periódicos brasileiros. As primeiras publicações datam do ano de 1995; somente a partir de 2002 o tema passa a ser abordado com maior frequência de publicações. Dentre as áreas de educação ou ensino, pesquisa e assistência, a área de educação em enfermagem é a que mais tem utilizado os recursos dessas tecnologias. ${ }^{18}$

Percebe-se, dessa forma, que os profissionais de enfermagem precisam ficar atentos às inovações pertinentes à sua prática, que visem ao benefício da profissão e ao cuidado ao cliente, visto que são inúmeras as formas de utilização e aproveitamento dos recursos tecnológicos para informação, comunicação e internet pela enfermagem em países desenvolvidos, porém ainda pouco exploradas na melhoria da organização e prática do cuidado pela enfermagem brasileira. ${ }^{18}$

Em 1998, já se apontava o Prontuário Eletrônico do Paciente (PEP), no Brasil, como uma tendência daquela época, alertando os enfermeiros que, se quisessem ter seus registros no PEP, teriam de se responsabilizar por isso. Supostamente, o PEP deveria contemplar o registro de todas as intervenções realizadas pela equipe multiprofissional ao paciente e demais registros inerentes ao tipo de documento. ${ }^{17}$

O PEP, ao utilizar informação integrada, possibilita em sua proposta a união de "todos os diferentes tipos de dados produzidos em variados formatos, em épocas diferentes, feitos por diferentes profissionais da equipe de saúde em distintos locais" 6:356 Assim, as informações sobre o histórico de saúde e doença do cliente registradas num PEP podem ser armazenadas e mantidas em rede informacional e, assim, ser compartilhadas entre os profissionais e serviços da saúde, através de sistemas de informação, de acordo com o direito e possibilidade de acesso de cada um.

É comum que os pacientes mudem de ou alternem entre os serviços de saúde, sejam públicos, sejam privados. Assim, um PEP disponível on-line com acesso em qualquer serviço de saúde, mediante uso da internet e, para a segurança do paciente, sob senha intransferível, seria de indubitável retorno em qualidade de informação para o cuidador e para o ser cuidado. O profissional - ser cuidador -, ao conhecer o histórico de saúde e doença do seu cliente - ser cuidado -, poderia atendê-lo de forma integrada com os diversos departamentos e 
especialidades em saúde. Seria possível interagir com os demais profissionais da enfermagem e saúde para a intervenção mais adequada ao cliente, tendo acesso interativo à informação e à imagem de exames realizados, a medicações em uso pelo cliente e ou prescritas por profissionais (que, por algum motivo, o cliente esteja em desuso).

Porém, questiona-se: Essa é uma possibilidade tangível? Tal possibilidade depende de investimentos financeiros por parte de profissionais autônomos, pelos órgãos públicos e particulares de saúde e, principalmente, de esforços dos profissionais envolvidos no processo. Indubitavelmente, a médio ou longo prazo, profissionais e pacientes ganharão em comodidade, agilidade, eficácia, eficiência e qualidade do cuidado com um serviço disponível em rede que possibilitaria a interação, associação e relação entre os seres cuidadores e seres cuidados.

\section{CONSIDERAÇÕES FINAIS}

Com base nas reflexões, ressaltamos que o trabalho dos enfermeiros é complexo, e gerenciar este trabalho em distintos ambientes, com prioridades e diversidade profissional que lhes competem, é extremamente difícil, dadas as lacunas e descontinuidades inerentes aos sistemas de cuidado em saúde. Os enfermeiros estão regularmente expostos a novas tecnologias, que necessitam ser integradas nos sistemas de prestação do cuidado, enquanto que as políticas e procedimentos que gerenciam o uso das tecnologias preexistentes exigem garantias e seu uso correto. Cada tecnologia adicionada ao processo de cuidar em enfermagem e aos sistemas organizacionais aumenta a complexidade do trabalho dos enfermeiros, porém, também potencializa a redução da sobrecarga de trabalho, melhorando a qualidade do cuidado e diminuindo os eventos adversos e erros. ${ }^{19}$

Deduzimos que conceber as TIs, contextualizar a realidade profissional e compreender a complexidade do cuidado em suas múltiplas dimensões é, para a enfermagem contemporânea, um desafio complexo. A inter-relação desses aspectos é uma possibilidade de inovar sua organização/gerência e prática de cuidado do outro, de forma a corresponder às necessidades de um mundo globalizado.

A enfermagem precisa progredir na área das TIs, visto que não avançou muito nos últimos anos, talvez por receio, insegurança, indecisão, imprecisão dos riscos e/ou benefícios em atrelar as tecnologias à prática do cuidado humano. $\mathrm{O}$ profissional de enfermagem precisa ser incitado e instigado a olhar de forma mais ampla, acurada, crítica e reflexiva a realidade que o cerca. A presença das tecnologias no cotidiano da enfermagem não pode ser negada, pois, diante da realidade tecnológica disponível, os profissionais precisam pensar e refletir sobre: Como utilizar, conviver e viver saudavelmente com TIs no cotidiano de cuidado dos profissionais de enfermagem em ambientes de saúde? De que forma essas tecnologias podem facilitar e promover relações de cuidado entre os profissionais e pacientes no ambiente/cenário da saúde? Como viabilizar relações humanas mais saudáveis com o uso dessas tecnologias? Como as tecnologias podem apoiar ou instrumentalizar a gerência do cuidado de enfermagem?

Ocorre em nosso contexto social a preocupação com o uso excessivo das tecnologias para o estabelecimento de relações entre os seres humanos, demasiadamente virtuais, por meio de máquinas e equipamentos, que, consequentemente, podem maximizar o distanciamento entre os seres de relação, o contato pessoa-pessoa e, por fim, banir as relações sociais. Dessa forma, o contato e a convivência entre os seres humanos não devem ser ignorados, pois são percebidos como necessários e importantes para as relações de cuidado saudáveis e construtivas. A importância da tecnologia não pode ser maior que a do ser humano. O uso da tecnologia deve estar atrelado à satisfação de necessidades humanas, e o ser humano, seu criador, deve buscar aperfeiçoá-la para o seu beneficio e da coletividade, como forma de cuidado.

\section{REFERÊNCIAS}

1. Royal College of Nursing. E-health: putting information at the heart of nursing care London [página na Internet]. 2006 [acesso 2007 Jun 28]; 1-8. Disponível em: http://www.rcn.org.uk/information

2. Morin E. Os sete saberes necessários à educação do futuro. $11^{\mathrm{a}}$ ed. São Paulo (SP): Cortez; 2006.

3. Morin E, Kern AB. Terra-Pátria. $5^{\mathrm{a}}$ ed. Porto Alegre (RS): Sulina; 2005.

4. Morin E. A cabeça bem-feita: repensar a reforma, reformar o pensamento. Rio de Janeiro (RJ): Bertrand Brasil; 2000.

5. Sasso GTMD; Martins CR. Tecnologia: definições e reflexões para a prática em saúde e enfermagem. Texto Contexto Enferm. 2008 Jan-Mar; 17(1):11-2.

6. Marin HF, Cunha ICKO. Perspectivas atuais da Informática em Enfermagem. Rev Bras Enferm. 2006 Maio-Jun; 59(3):354-7. 
7. Lemos A. Cidade-Ciborgue: a cidade na cibercultura Galáxia. 2004 Out; 4(8):129-48.

8. Lévy P. As tecnologias da inteligência: o futuro do pensamento na era da informática. Rio de Janeiro (RJ): Editora 34; 1993.

9. Sasso GTMD. A concepção do enfermeiro na produção tecnológica informatizada para o ensino/ aprendizagem em reanimação cárdio-respiratória [tese]. Florianópolis (SC): Universidade Federal de Santa Catarina. Programa de Pós-Graduação em Enfermagem; 2001.

10. Oliveira FR, Pizzi F, Gonçalves MS. Ciborgue: humano e comunicação. Rev Ghrebh [online]. 2004 Nov; 6: [acesso 2008 Nov 20]. Disponível em: http:/ / revista.cisc.org.br/ghrebh6/artigos/06fatima.htm

11. Potter P, Boxerman S, Wolf L, Marshall J, Grayson D, Sledge J, Evanoff B. Mapping the nursing process: a new approach for understanding the work of nursing. J Nurs Adm. 2004; 34:101-9.

12. Sipe M, Marthinsen J, Baker J, Harris J, Opperman J. Using technology to improve patient care. Nurs Outlook. 2003; 51: s35-6.
13. Bettinelli LA. A solidariedade no cuidado: dimensão e sentido da vida. Florianópolis (SC): UFSC/PEN; 2002.

14. Meyer DE. Como conciliar humanização e tecnologia na formação de enfermeiras/os? Rev Bras Enferm. 2002 Mar-Abr; 55(2):189-95.

15. Falcón GS, Erdmann AL. La ética y la técnica contemporánea: Implicaciones en el Área de la Salud. An Fac Med Lima. 2005; 66(1):71-9.

16. Andrade JS, Vieira MJ. Prática assistencial de enfermagem: problemas, perspectivas e necessidade de sistematização. Rev Bras Enferm. 2005 Maio-Jun; 58(3):261-5.

17. Marin HF, Granitoff N. Informática em enfermagem: uma experiência. Acta Paul Enf. 1998; 11(Esp):42-5.

18. Santos SGF, Marques IR. Uso dos recursos de Internet na Enfermagem: uma revisão. Rev Bras Enferm. 2006 Mar-Abr; 59(2):212-6.

19. Zuzelo PR, Gettis C, Hansell AW, Thomas L. Describing the influence of technologies on registered nurses' work. Clin Nurse Spec. 2008, 22(3):132-40. 